\title{
Dilatação esofágica endoscópica associada ao uso de triancinolona intramural em cadela com estenose de esôfago após ovariohisterectomia eletiva
}

\author{
Endoscopic esophageal dilation associated with intramural triamcinolone in a bich with \\ esophageal strictures after elective ovariohisterectomy
}

\author{
Marília Teresa de Oliveira ${ }^{\mathrm{I}}$ Anelise Bonilla Trindade ${ }^{\mathrm{II}}$ Fernando Wiecheteck de Souza ${ }^{\mathrm{I}}$ \\ Fabiola Dalmolin ${ }^{I}$ Saulo Tadeu Lemos Pinto Filho ${ }^{I}$ Bernardo Schmitt ${ }^{\mathrm{I}}$ Paula Cristina Basso ${ }^{\mathrm{I}}$ \\ João Pedro Scussel Feranti ${ }^{\mathrm{I}}$ Maurício Veloso Brun ${ }^{{ }^{*}}$
} \begin{abstract}
A estenose esofágica adquirida ocorre normalmente
em função de uma esofagite severa, resultando em processo RESUMO cicatricial com proliferação de tecido fibroso. Entre as causas, encontra-se o refluxo gastroesofágico em animais anestesiados. $O$ presente trabalho objetiva relatar o tratamento de estenose esofágica em uma cadela por dilatações luminais endoscópicas, associadas ao uso de triancinolona intralesional. Um canino, $S R D$, foi atendido com a queixa de regurgitação e emagrecimento progressivo. Esses sinais surgiram após a realização de $\mathrm{OVH}$ videolaparoscópica eletiva, durante a qual a paciente apresentou regurgitação no transoperatório. Com base no exame clínico e nos achados endoscópicos, foi possível confirmar o diagnóstico de estenose no terço final do esôfago cervical. Após o diagnóstico definitivo, iniciou-se o tratamento com dilatações esofágicas intraluminais endoscópicas e administração intralesional de triancinolona. Esse procedimento foi repetido uma vez por semana, durante quatro semanas. A quinta dilatação ocorreu após 14 dias da quarta dilatação. O paciente teve alta hospitalar após sete dias da quinta e última seção de dilatação esofágica e aplicação de triancinolona, não apresentando mais episódios de regurgitação, mesmo ao se alimentar com ração seca sem plano inclinado. Conclui-se que as dilatações esofágicas associadas ao uso de triancinolona intralesional foram efetivas no tratamento de estenose de esôfago no presente caso.
\end{abstract}

Palavras-chave: endoscopia, regurgitação, corticosteroide.

\section{ABSTRACT}

The acquired esophageal stenosis usually occurs due to a severe esophagitis, resulting in the healing process with proliferation off ibrous tissue. Is gastroesophageal refluxin anesthetized animals among the reasons of this disease. This paper reports a case of esophageal luminal endoscopic dilations associated with intralesional triamcinolone in a bitch with esophageal strictures. A mongrel canines hows regurgitation and progressive weight loss. These signs appeared after elective laparoscopic $\mathrm{OVH}$ performed, where the patient had part of regurgitation during surgery. Based on clinical and endoscopic findings it was possible to diagnostic stenosis in the final third of the cervical esophagus. The treatment was done with esophageal dilations and intraluminnal triamcinolone administration. This procedure was repeated once a week for four weeks. The $15^{\text {th }}$ dilatation occurred 14 days after the $14^{\text {th }}$. The patient was discharged after seven days of the final dilatation with no more regurgitation, even when feeding dry kibble within o incline. We concluded that the esophageal dilation associated with in tralesional triamcinolone were effective esophageal strictures treatment in this case.

Key words: endoscopy, regurgitation, corticosteroid.

A estenose esofágica adquirida ocorre normalmente em função de esofagite severa, resultando em processo cicatricial com proliferação de tecido fibroso (GUILFORD \& STROMBECK, 1996), o qual compromete, por sua vez, o lúmen do órgão (SELLON \& WILLARD, 2003). O refluxo gastroesofágico em animais anestesiados encontra-se entre as causas mais comuns de esofagite (HARAI et al., 1995). Os sinais clínicos associados dependem da localização e do diâmetro da estenose (GUILFORD \& STROMBECK, 1996), sendo a regurgitação o mais evidente (SELLON \& WILLARD, 2003). O tratamento pode ser realizado por meio de dilatações esofágicas, aplicação local de corticoides (BROOR et al., 1993) ou correção cirúrgica (GUALTIERI, 2001).

'Laboratório de Cirurgia Experimental, Universidade Federal de Santa Maria (UFSM), Avenida Roraima,1000, 97105-900, Santa Maria, RS, Brasil. E-mail: mauriciovelosobrun@hotmail.com.*Autor para correspondência.

IIMédica Veterinária autônoma, Porto Alegre, RS, Brasil. 
Em virtude da escassez de estudos referentes ao tratamento detalhado de lesões esofágicas estenosantes em cães, o presente trabalho objetiva relatar um caso de dilatações esofágicas por endoscópicas associadas ao uso de triancinolona intralesional em cadela com estenose de esôfago.

Um canino, adulto, SRD, fêmea, foi atendido com a queixa de regurgitação e emagrecimento progressivo, sem perda do apetite. Estes sinais surgiram aproximadamente 15 dias após a realização de OVH videolaparoscópica eletiva, ocasião na qual a paciente havia apresentado quadro de regurgitação no transoperatório.

Com base no exame clínico e achados endoscópicos, já que o esofagograma contrastado não foi conclusivo, confirmou-se o diagnóstico de estenose no terço final do esôfago cervical. Após o diagnóstico definitivo, iniciou-se o tratamento com dilatações esofágicas e administração intramural de triancinolona (40mg). Para aplicação do fármaco, foi utilizada uma agulha espinhal de $22 \mathrm{~cm}$ de comprimento, introduzida no interior de traqueotubo 7,5. Após a introdução do conjunto, sob visão, a agulha era exposta para administração da medicação. Esse procedimento foi repetido uma vez por semana, durante quatro semanas. A quinta dilatação ocorreu após 14 dias da quarta.

A localização endoscópica da estenose permitiu a utilização de tubos endotraqueais adaptados para realização das dilatações, a partir da distensão dos seus cuffs preenchidos com $\mathrm{NaCl}$ 0,9\%. Na primeira seção, utilizou-se endoscópio flexível, sendo realizadas três dilatações, com tempo de duração de dois minutos cada, tempo esse respeitado em todas as dilatações subsequentes. Primeiramente, foi utilizado traqueotubo 4 e a última dilatação do dia foi realizada com traqueotubo 5 (Figura 1 A). Realizou-se ainda gastrostomia videoassistida com a aplicação de Foley, sendo que a paciente removeu a sonda por três vezes durante o período de seis dias e, em duas ocasiões, a sonda foi reposicionada via cirurgia convencional.

A partir da segunda semana, empregouse endoscópio rígido $(10 \mathrm{~mm})$, sendo realizadas quatro dilatações por seção, iniciando com sonda endotraqueal 5 e as duas últimas dilatações da segunda seção permitiram a passagem da sonda 6 (Figura 1 B). Na terceira semana, as duas últimas dilatações foram realizadas com sonda endotraqueal 7 (Figura 1 C), permitindo a visualização do esôfago torácico cranial, até então não visualizado.

Um segundo ponto de estenose foi observado na quarta semana de tratamento, cranial ao ponto da estenose inicial, então foram realizadas duas dilatações com traqueotubo 8 em cada ponto de estenose e, posteriormente, foram realizadas duas dilatações com a sonda 9 (Figura 1 D). Já na última semana de tratamento, tentou-se a passagem do traqueotubo 9, sem sucesso. Então as duas primeiras dilatações foram realizadas com sonda 8,5, possibilitando a dilatação sequencial com sonda 10 (Figura $1 \mathrm{E}$ ).

A partir da terceira semana de tratamento, a paciente começou a ganhar massa corporal, sendo a alimentação realizada com ração líquida em plano inclinado. Teve alta hospitalar após sete dias da quinta e última dilatação esofágica, vindo a não apresentar mais episódios de regurgitação, mesmo ao se alimentar com ração seca sem plano inclinado. Após o período mínimo de 8 meses, o animal não apresentou sinais clínicos de recidiva.

Embora a estenose esofágica possa ser causada por qualquer lesão grave à mucosa (ADAMAMA-MORAITOU et al., 2002), seja por traumas, agentes químicos, térmicos ou infecciosos (HARAI et al., 1995), observa-se que a maioria dos casos ocorre por complicação de esofagite de refluxo após anestesia (ADAMAMA-MORAITOU et al., 2002; SELLON \& WILLARD, 2003), principalmente em fêmeas submetidas a ovariohisterectomia (OVH) (ADAMAMA-MORAITOU et al., 2002). Esses dados auxiliaram no diagnóstico da enfermidade, já que o animal se enquadrava nessas características.

Alguns autores descrevem que através do esofagograma é possível detectar estenoses longas e múltiplas (ZAWIE, 1989; WEYRAUCH \& WILLARD, 1998; HARAI et al., 1995), fato não observado no caso, sendo o diagnóstico definitivo possível somente por meio dos achados endoscópicos, corroborando JOHNSON \& SHERDING (1998) e SILVA (2010), que salientam a eficácia da técnica.

Dentre as opções de tratamento, optouse pelo método conservador, já que algumas características do esôfago cervical, como falta de revestimento seroso, vascularização sanguínea segmentar, ausência de distensão longitudinal e movimentação aumentam as possibilidades de complicações cirúrgicas nesse órgão (JOHNSON \& SHERDING, 1998). Na medicina humana, o tratamento de escolha é a dilatação esofágica (PEREIRA-LIMA et al., 1999). Considerando que cada procedimento de dilatação realizado acarreta nova lesão na região estenosada (ULMAN \& MUTAF, 1988), podendo levar ao restabelecimento da doença, optou-se pelas dilatações seguidas de aplicação intralesional de triancinolona. Esse é o corticoide de escolha para lesões estenosantes de difícil tratamento, 


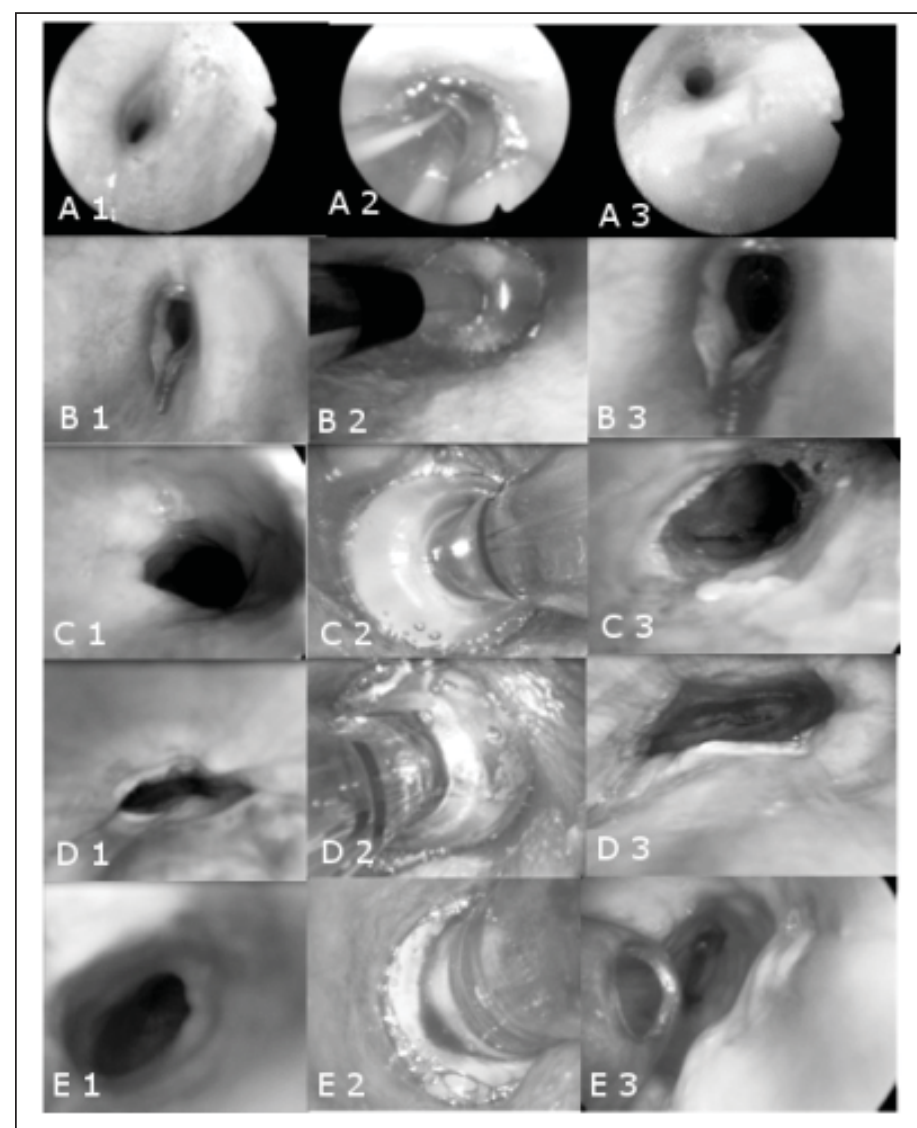

Figura 1 - Representação das cinco seções de tratamento (A,B,C,D,E) com dilatações esofágicas seguidas de aplicação de triancinolona intralesional, em canino, fêmea, SRD, adulto. A1 -Ponto de estenose esofágica; A2 - Primeira dilatação; A3 - Esôfago após a primeira seção de tratamento. B1 - Ponto de estenose esofágica; B2 - Segunda dilatação; B3 - Esôfago após a segunda seção de tratamento. C1 Ponto de estenose esofágica; C2 - Terceira dilatação; C3 - Esôfago após a terceira seção de tratamento. D1 - Ponto de estenose esofágica; D2 - Quarta dilatação; D3 - Esôfago após a quarta seção de tratamento. E1 - Ponto de estenose esofágica; E2 - Quinta dilatação; E3 - Esôfago após a quinta e última seção de tratamento.

já que interfere no "crosslink” do colágeno, o qual resulta na contração da cicatriz (LEE, 1995), além de diminuir a resposta inflamatória, interferir na síntese de colágeno, fibrose e cicatrização crônica (BROOR et al., 1993).

Assim como em um estudo desenvolvido em humanos, no qual foram realizadas dilatações esofágicas e múltiplas aplicações de triancinolona local, observou-se aumento significativo do diâmetro esofágico (CAMARGO et al., 2003), o qual permitiu ao paciente retornar à alimentação sólida, sem a necessidade de plano inclinado e sem apresentar sinais clínicos.Cabe salientar que a situação foi obtida mesmo sem a utilização de dilatador específico, adaptando-se duas sondas endotraqueais unidas para alcançar a lesão.
Conclui-se que as dilatações esofágicas associadas ao uso de triancinolona intralesional foram efetivas no tratamento de estenose de esôfago no presente caso.

\section{COMITÊ DE ÉTICA E BIOSSEGURANÇA}

Declaração dos autores - O relato foi executado com animal atendido na rotina do Hospital Veterinário Universitário UFSM - e não foi parte de qualquer projeto, sendo apenas um relato de caso clínico. Os autores ficam à disposição para quaisquer esclarecimentos futuros sobre o trabalho.

\section{REFERÊNCIAS}

ADAMAMA-MORAITOU, K.K. et al. Benign esophageal stricture in dogand cat: a retrospective study of 20 cases. Canadian Journal of Veterinary Research, v.66, p.55-59, 2002. Disponível

Ciência Rural, v.43, n.9, set, 2013. 
em: <http://www.ncbi.nlm.nih.gov/pmc/articles/PMC226983/>. Acesso em: 24 nov. 2012

BROOR, S.L., et al. Long term results of endoscopic dilation for corrosive esophageal strictures. International Journal of Gastroenterology and Hepatology, v.34, p.1498-501, 1993. Disponível em: <http://www.ncbi.nlm.nih.gov/pmc/articles/ PMC1374409/>. Acesso em: 24 nov. 2012.

CAMARGO, M.A. et al. O uso de corticoesteróides após dilatação esofágica em pacientes portadores de estenose por substâncias corrosivas - estudo prospectivo, randomizado e duplo-cego. Revista da Associação Médica Brasileira, v.49, n.3, p.286-92, 2003. Disponível em: <http://www.scielo.br/scielo. php?script=sci_arttext\&pid=S0104-42302003000300033\&lng= en\&nrm=iso>. Acesso em: 18 nov. 2012. doi: 10.1590/S010442302003000300033

GUALTIERI, M. Esophagoscopy. The Veterinary Clinics of North America: Small Animal Practice. v. 31, p. 605-630, 2001. Disponível em: <http://www.mendeley.com/catalog/esophagitisesophageal-strictures/>. Acesso em: 24 nov. 2012.

GUILFORD, W.G.; STROMBECK, D.R. Diseases of swallowing. In: GUILFORD, W.G., et al. Small animal gastroenterology. 3.ed. Philadelphia: Saunders, 1996. p.211-238.

HARAI, B.H. et al. Endoscopically guided balloon dilatation of benign esophageal strictures in 6 cats and 7dogs. Journal of Veterinary Internal Medicine, v.9, p.332-335, 1995. Disponível em: <http://www.ncbi.nlm.nih.gov/pubmed/8531179>. Acesso em: 24 nov. 2012

JOHNSON, S.E.; SHERDING, R.G. Doenças do esôfago e distúrbiosde deglutição. In: BIRCHARD, S.J.; SHERDING, R.G. Manual Saunders: clínica de pequenos animais. São Paulo: Roca, 1998. P.709-725.
LEE, M. Preliminary experience with endoscopic intralesional steroid injection therapy for refractory upper gastrointestinal strictures. Gastrointestinal Endoscopy, v.41, p.598-601, 1995. Disponível em: <http://www.ncbi.nlm.nih.gov/pubmed/7672557>. Acesso em: 24 nov. 2012.

PEREIRA-LIMA, J.C. et al. Endoscopic dilation of benign esophageal strictures: report on 1043 procedures. American Journal of Gastroenterology, v.94, p.1497-500, 1999. Disponível em: <http://www.ncbi.nlm.nih.gov/pubmed/10364013>. Acesso em: 24 nov. 2012

SELLON, R.K.; WILLARD, M.D. Esophagitis and esophageal strictures. Veterinary Clinics of North America: Small Animal Practice, v.33, n.5, p.945-967, 2003. Disponível em: <http:// www.mendeley.com/catalog/esophagitis-esophageal-strictures/>. Acesso em: 24 nov. 2012.

SILVA, E.C.S. Diagnóstico e tratamento da estenose esofágica pela via endoscópica em cão: relato de caso. Ciência Animal Brasileira, v.11, n.2, p.465-470, 2010. Disponível em: <http:// www.revistas.ufg.br/index.php/vet/article/view/4120>. Acesso em: 28 nov. 2012. doi: 10.526/cab.v11i2.4120.

ULMAN, I; MUTAF, O. A critique of systemic steroids in the management of caustic esophageal burns in children. European Journal of Pediatric Surgery, v.8, p.71-74, 1998. Disponível em: <http://www.ncbi.nlm.nih.gov/pubmed/9617603>. Acesso em: 18 nov. 2012.

WEYRAUCH, E.A.; WILLARD, M.D. Esophagitis and benign esophageal strictures. Compendium on Continuing Education for the Practicing Veterinarian, v.20, p.203-211, 1998. Disponível em: <http://openagricola.nal.usda.gov/Record/ IND21513948>. Acesso em: 24 nov. 2012.

ZAWIE, D.A. Esophageal strictures. In: KIRK, R.W.; BONAGURA, J.D. Current veterinary therapy. X. Small animal practice. Philadelphia: Saunders, 1989. p.904-906. 